\title{
Usefulness of three-dimensional digital image analysis for objective evaluation of the efficacy of non-facial port-wine stain treatment with large spot $532 \mathrm{~nm}$ laser
}

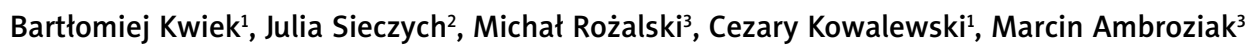 \\ ${ }^{1}$ Department of Dermatology and Immunodermatology, Medical University of Warsaw, Warsaw, Poland \\ ${ }^{2}$ Department of Dermatology and Venereology, Medical University of Warsaw, Warsaw, Poland \\ ${ }^{3}$ Clinic Ambroziak, Warsaw, Poland
}

Adv Dermatol Allergol 2020; XXXVII (4): 572-578

DOI: https://doi.org/10.5114/ada.2019.83520

\begin{abstract}
Introduction: New devices such as the large spot KTP laser are being introduced for the treatment of port-wine stains (PWS).

Aim: To assess the efficacy of the large spot $532 \mathrm{~nm}$ laser for non-facial PWS with 3D image analysis and compare it with subjective evaluation.

Material and methods: Twenty PWS were photographed with a 3D photo unit before and after $532 \mathrm{~nm}$ large spot KTP laser treatment. Fifteen lesions were previously treated by different devices and five were not. Objective analysis of percentage improvement based on a 3D digital assessment of combined color and area improvement was performed and rates of improvement were determined as well as subjective evaluation of before and after images by a physician on a 5 -grade scale.

Results: Mean objective response was $57.0 \%$. A poor response was observed in $5 \%$ with the objective method and with no patient with the subjective method. A moderate response was achieved by $25 \%$ and $30 \%$ with the objective and subjective assessment respectively. A significant response was obtained by $55 \%$ objectively and $10 \%$ subjectively. $75-100 \%$ was achieved by $15 \%$ and $60 \%$ in the objective and subjective analysis respectively. The two methods significantly correlated with each other but the average subjective improvement rates were higher than the objective rates.

Conclusions: Both objective and subjective analysis indicated that the large spot $532 \mathrm{~nm}$ laser is highly effective in the treatment of the neck and trunk. 3D color and area objective analysis provides an accurate tool to measure the efficacy of PWS treatment.
\end{abstract}

Key words: port-wine stain, KTP, pulse dye laser, 3D, laser, port-wine stain.

\section{Introduction}

Port-wine stain (PWS) are a common type of cutaneous capillary malformations that affect $0.3 \%$ of newborns. They are related to segmental mosaicism and they can occur in all body regions following the patterns of vascular embryogenesis [1-4]. Lasers are the first line option for the treatment of PWS. There are several types of devices available for PWS treatment, including pulsedye laser (PDL), intense pulse light (IPL), small and large spot $532 \mathrm{~nm}$ KTP (potassium-titanyl-phosphate) laser, and $1064 \mathrm{~nm}$ neodymium-doped yttrium aluminum garnet (Nd:YAG) laser. Others such as alexandrite $755 \mathrm{~nm}$ or diode 800-983 nm lasers are less commonly used and argon, copper and krypton lasers are currently not recommended because of the poor safety-to-efficacy profile [5]. Recently photodynamic therapy (PDT) has shown promising results for the treatment of PWS in Chinese patients [6].

Beside PDT, all these devices use hemoglobin as a photon absorber to destroy affected vessels. The majority of patients respond to treatment, but clearance is hardly ever achieved. The degree of improvement depends on several factors including the type of device used and PWS localization. Although PDL is recognized as the most effective, the large spot $532 \mathrm{~nm}$ KTP laser has recently been shown to be

Address for correspondence: Bartłomiej Kwiek MD, Department of Dermatology and Immunodermatology, Medical University of Warsaw, 82 A Koszykowa St, 02-008 Warsaw, Poland, phone/fax: +48 2250213 13, e-mail: bartlomiej@kwiek-dermatolog.pl Received: 30.01.2019, accepted: 15.02.2019. 
highly valuable for facial PWS. This device is based on a frequency-doubled, $532 \mathrm{~nm} \mathrm{Nd:YAG} \mathrm{laser} \mathrm{and}$ is characterized by a large spot (up to $12 \mathrm{~mm}$ ), short pulse and contact cooling. Earlier reports on $532 \mathrm{~nm}$ KTP lasers were performed with tools that generate a small spot size with up to 2-4 $\mathrm{mm}$ diameter and/or long pulse width [7-10]. Larger diameter of the laser beam is crucial for the green light $(532 \mathrm{~nm}$ ) to sufficiently penetrate deep into the dermis or even the subcutaneous fat so as to close the dilatated vessel, as was shown in both vivo and in vitro studies [11, 12].

The assessment of the efficacy of PWS treatment is usually based on the subjective methods of physicians or the patient's judgment. This makes comparison of the results of different studies difficult. Several objective methods of analysis of two-dimensional (2D) digital or digitalized photography were proposed, but they do not consider the complex shape three-dimensional (3D) nature of most PWS. Other devices based on colorimetric methods assess only the color of the lesion, neglecting its area [13-17]. Analyzing combined measurement of an average color of the lesion and area measured with $3 \mathrm{D}$ image analysis has proven its value in our previous reports on facial PWS $[5,18]$. In these reports we used a commercially available tool for taking 3D face and body images. Here we propose to use this tool for the objective assessment of PWS located on the neck and trunk.

\section{Aim}

The aim of the study was to assess the efficacy of the large spot $532 \mathrm{~nm}$ laser in the treatment of neck and truncal PWS using both 3D color and area analysis and subjective judgment of improvement made by the physician.

\section{Material and methods}

The study was approved by the Institutional Review Board. We performed a one-center, retrospective study, which included patients with both previously treated (15) and untreated (5) PWS who came to our clinic between January 2014 and June 2018 and who were treated by two physicians (B.K. and M.R.). The PDL was previously used on 4 of these patients, IPL also on four, large spot KTP on 3 patients, and small spot KTP and argon laser were both used on 1 patient. Three patients were treated with an unknown laser. Only patients who had 3D photographs performed before and after and had at least one single procedure were included in the study. All patients were Caucasians (13 females and 5 males), aged from 12 to 66 (mean \pm SD: $37 \pm 14.3$ ). Most patients (13) had skin phototype II according to the Fitzpatrick scale and 1 had phototype I and 4 patients had phototype III. Patients currently tanned or with a history of sun and/or ultraviolet exposure within 1 month prior to the procedure were asked to come after more than 4 weeks of careful sun protection. All patients were treated with a large spot, frequency-doubled, $532 \mathrm{~nm} \mathrm{Nd:YAG} \mathrm{(KTP)} \mathrm{laser} \mathrm{with} 5^{\circ} \mathrm{C}$ contact cooling provided by sapphire glass (ExcelV; Cutera Inc, Brisbane, CA, USA). A variable setting was used with the fluencies ranging from 7 to $9 \mathrm{~J} / \mathrm{cm}^{2}$, pulse duration ranging from 6 to $9 \mathrm{~ms}$ and spot size ranging from 6 to $9 \mathrm{~mm}$ according to the judgment of the physician. The largest available spot size for the preset fluence and time was preferable. Local anesthetic (tetracaine and lidocaine ointment for 30 min prior to the procedure) was used in 1 adolescent patient. Cooling with a cold pack was used for 20-30 min after each procedure. Patients were asked to use a post-treatment emollient for 7 days, avoid sun exposure and use topical preparations with sun protection factor $50+$. The minimal interval between treatments was 4 weeks.

3D images were taken with the Vectra XT (Canfield Scientific, NJ, USA) in standardized conditions according to the manufacturer's guidelines for facial and truncal images and analyzed as described previously [18]. Thirteen neck and seven truncal lesions were assessed. Selected surface area $\left(\mathrm{cm}^{2}\right)$ and selected area average color (described with $L^{*} a^{*} b^{*}$ coordinates) were used for further analysis. Whenever possible, healthy skin of a symmetric area served as a control for color evaluation. In other cases (e.g. lesion covering two sides of the neck), skin adjacent to the lesion was used. The difference between the color of the lesion and healthy skin $(\Delta T)$ was calculated according to the following equation $[14,16]$ :

$$
\Delta T=\left[\left(\Delta \mathrm{L}^{*}\right)^{2}+\left(\Delta \mathrm{a}^{\star}\right)^{2}+\left(\Delta \mathrm{b}^{\star}\right)^{2}\right]^{1 / 2} .
$$

Table 1. Comparison of two systems of PWS treatment evaluation

\begin{tabular}{|c|c|c|c|}
\hline \multicolumn{2}{|c|}{ Commonly used subjective physician assessment based on grades } & \multicolumn{2}{|c|}{ Objective 3D digital image analysis based on rates } \\
\hline Description & Improvement (\%) & Description & $\mathrm{GCE}_{\max }$ of minimum (\%) \\
\hline Failure & $\leq 0$ & Lack of GCE25 & $<25$ \\
\hline Mild/poor & $1-25$ & & \\
\hline Moderate & $2-50$ & GCE25 & $\geq 25$ \\
\hline Good/significant & $51-75$ & GCE50 & $\geq 50$ \\
\hline \multirow[t]{2}{*}{ Excellent/cured } & $76-100$ & GCE75 & $\geq 75$ \\
\hline & & GCE90 & $\geq 90$ \\
\hline
\end{tabular}




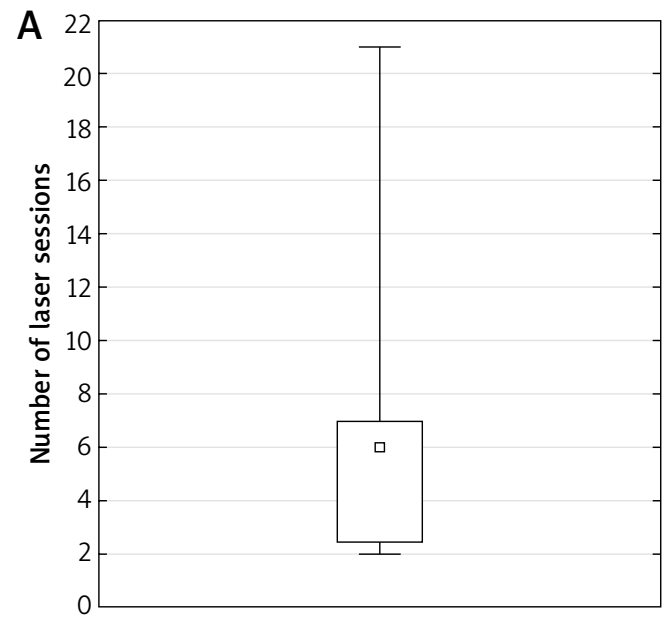

口Median $\square$ 25-75\% I Min.-max.
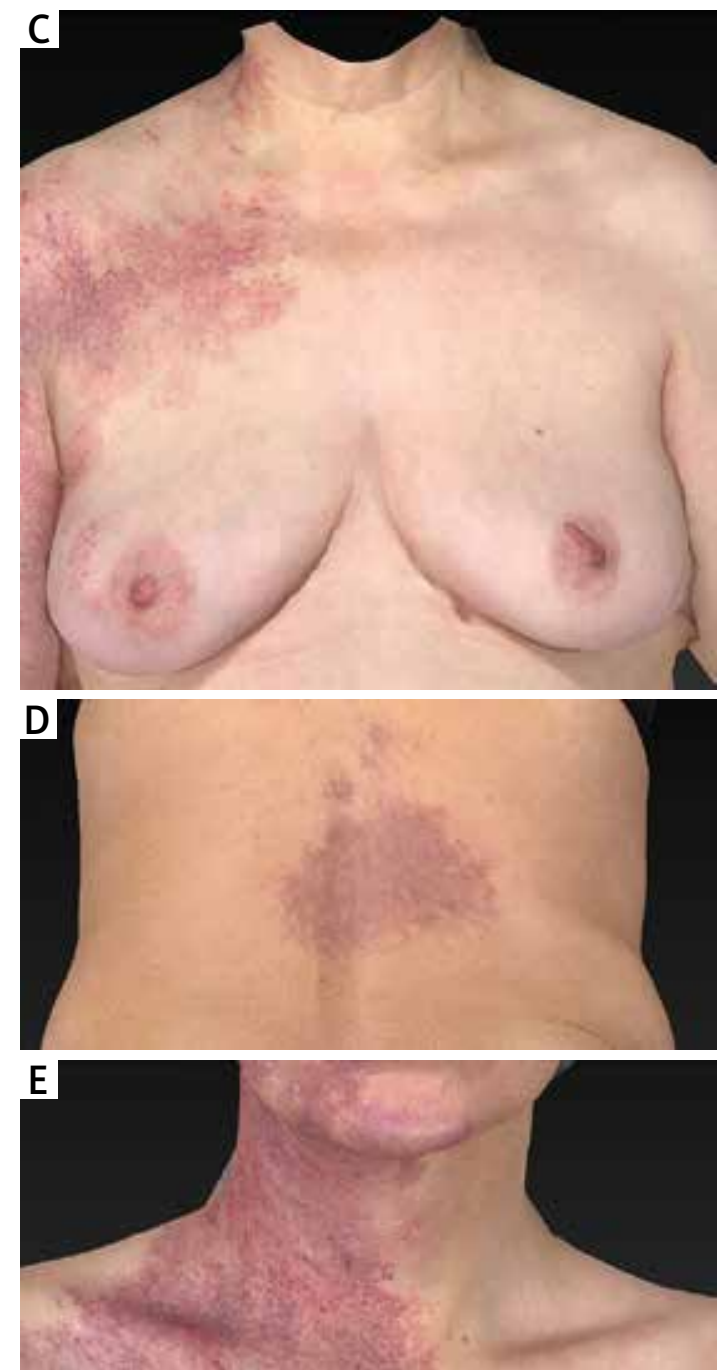

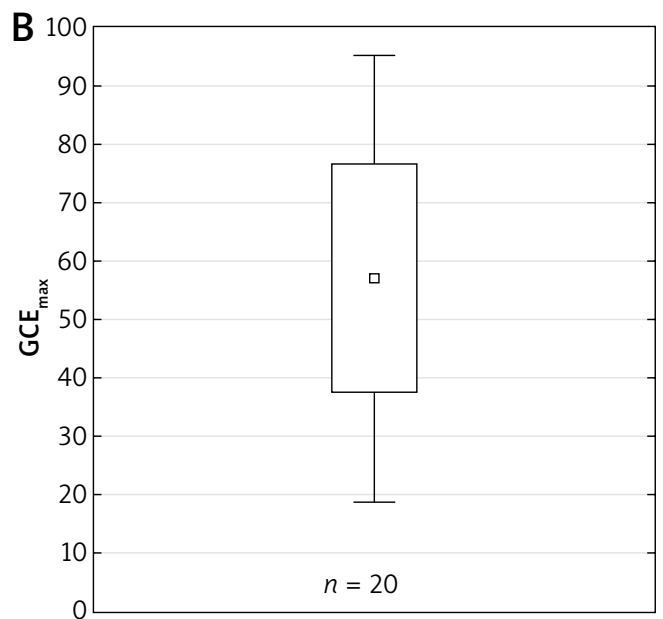

口Median $\square$ Mean \pm SD I Mean $\pm 1.98^{\star}$ SD
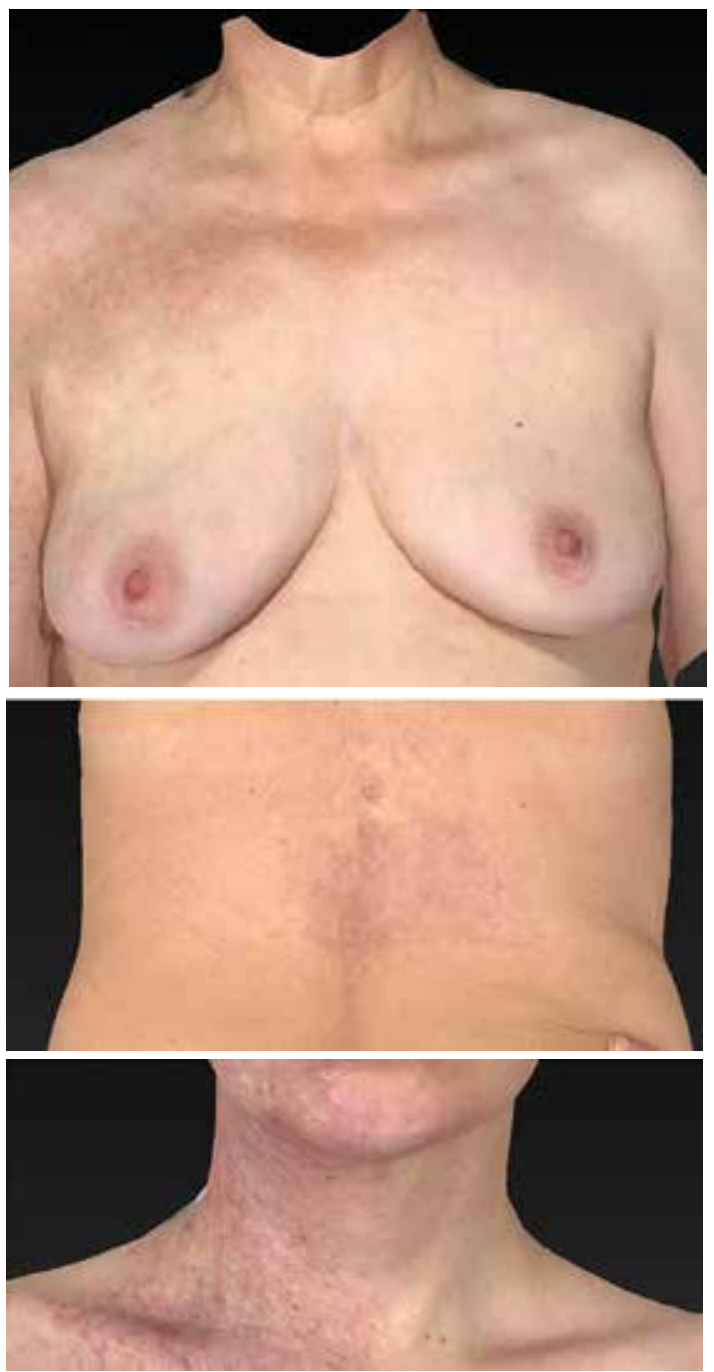

Figure 1. A - Number of laser sessions per patient. B - Maximal improvement (\%) achieved in 20 patients defined as maximal global clearance effect $\left(\mathrm{GCE}_{\max }\right)$. C-E - Before and after images (flattened 3D images) of three different PWS that were rated as $75-100 \%$ improvement ('cured' grade) subjectively. However, they are differently classified with the objective 3D measurement of the area and color. C - Objective result of the treatment (GCE ${ }_{\max }$ ) of the PWS located on the chest (trunk) is $88.0 \%$. D - GCE $\mathrm{max}_{\max }$ of the PWS located on the back is $66.6 \%$. E- GCE max $_{\text {me }}$ of the PWS located on the neck is $50.7 \%$ 
A

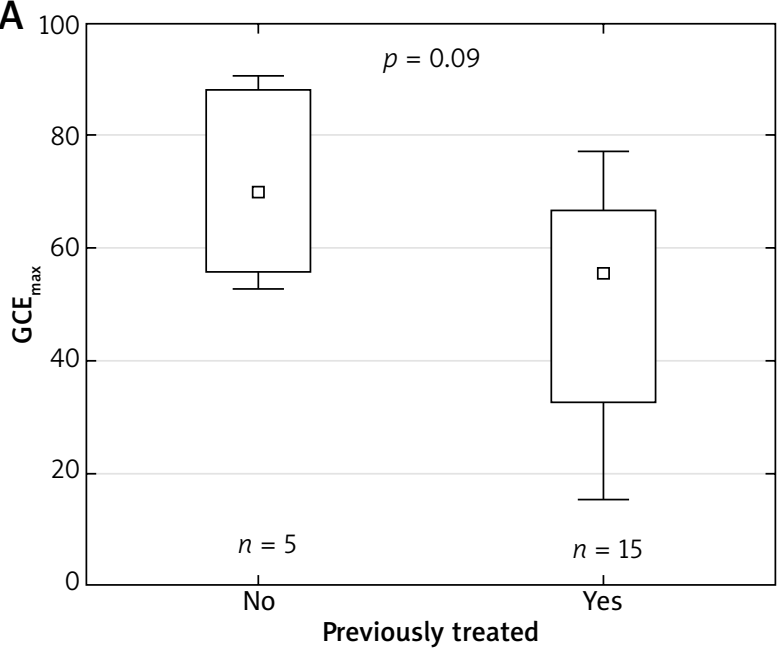

B

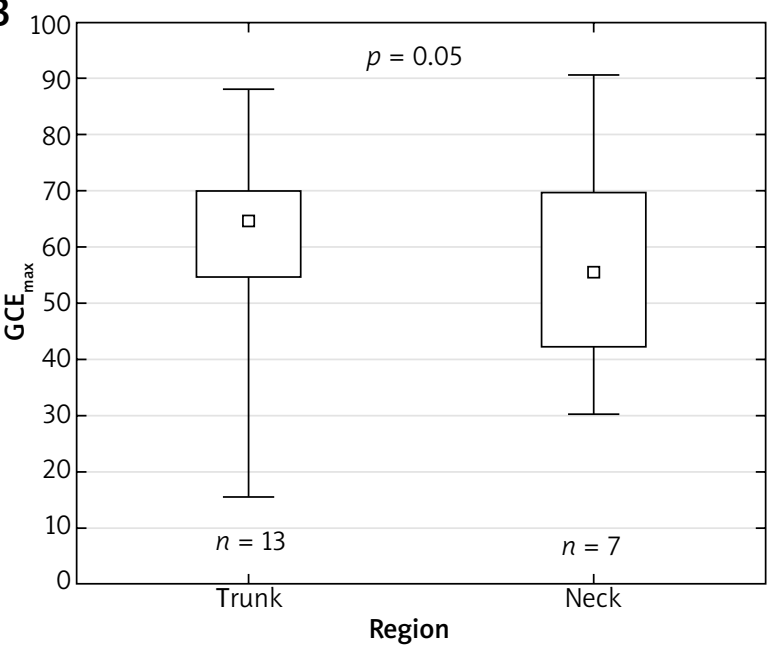

aMedian $\square$ 25-75\% I Min.-max.

Figure 2. A - Comparison of maximal improvement defined as maximal global clearance effect (GCE ${ }_{\max }$ ) between patients previously untreated and previously treated. $\mathbf{B}$ - Comparison of $\mathrm{GCE}_{\max }$ between PWS located on trunk and neck

To establish the improvement of the color of the lesion after treatment, the clearance effect (CE) was calculated as follows:

$C E=\left(1-\Delta T^{\text {after the treatment }} / \Delta T^{\text {before the treatment }}\right) \times 100 \%$.

Reduction of the area (A\%) of the lesion was calculated as the percentage difference between the area (A) before and after the treatment:

$A \%=\left(1-A^{\text {after the treatment }} / A^{\text {before the treatment }}\right) \times 100 \%$.

Finally, to combine the $A \%$ with the CE the, global CE (GCE) was calculated as follows:

$\mathrm{GCE}(\%)=\mathrm{A} \% \times 100+[(100-\mathrm{A} \%) \times \mathrm{CE})] / 100$.

Maximal GCE observed throughout the treatment of the patient was defined as GCE max . The rates of the patients achieving GCE ${ }_{\max }$ of minimum 25\% (GCE 25), 50\% (GCE 50), 75\% (GCE 75) and 90\% (GCE 90) during the treatment were calculated (Table 1). For safety evaluation, patients were asked to report the presence and longevity of erythema, edema and bruises as well as the occurrence of blistering and/or crusting or secondary infections. Skin was assessed for the presence of scars on each visit. Subjective evaluation was performed using flattened (3D into 2D) images from 0, 45, and 90. A five-grade scale of improvement was used: 0 (no improvement ), 1-25\% (mild), 26-50\% (moderate), 51-75\% (significant), 76-100\% (cured) (Table 1). For some comparisons with objective analysis, an additional threshold point of subjectively assessed $\geq 90 \%$ improvement was used. Subjective analysis was performed by two physicians independently (JS, MR). Whenever there were discrepancies between their grading, the images were reevaluated until a consensus was achieved. This happened in two out of 20 cases.

\section{Statistical analysis}

Statistical analysis was performed with Statistica 12.0 software (StatSoft, USA). Quantitative variables were characterized with mean, standard deviation or median, quartiles and range after testing normality with the Shapiro-Wilk test. Significance of differences between two groups of variables was tested with the Mann-Whitney test. Student's t-test was used for comparison between two related groups. Correlations were calculated with Spearman's test. Intergroup comparisons of discrete variable distributions were carried out with Pearson's $\chi^{2}$ test. All $p$-values $<0.05$ were considered to be statistically significant.

\section{Results}

\section{Efficacy}

Median number of treatment sessions to obtain a maximal response was 6 (range: 2-21) (Figure 1 A). Mean maximal improvement achieved during the treatment assessed objectively $\left(\mathrm{GCE}_{\max }\right)$ was $57 \%(n=20)$ (Figure $1 \mathrm{~B}$ ). Median $\mathrm{GCE}_{\max }$ in previously untreated patients was higher (69.9\%) than in previously treated patients (55.5\%) (Figure $2 \mathrm{~A}$ ). There was no significant difference in the response between truncal PWS and those located on the neck (Figure 2 B). Objectively assessed improvement $\left(\mathrm{GCE}_{\max }\right)$ significantly correlated with subjective grades (Figure $3 \mathrm{~A}$ ). To further analyze the correlation between these two methods, we used a response rate analysis employing objectively measured GCE 25, GCE 50, GCE 75, GCE 90 percentage response rates and similar rates calculated from subjective analysis (Figure 4). Im- 
A
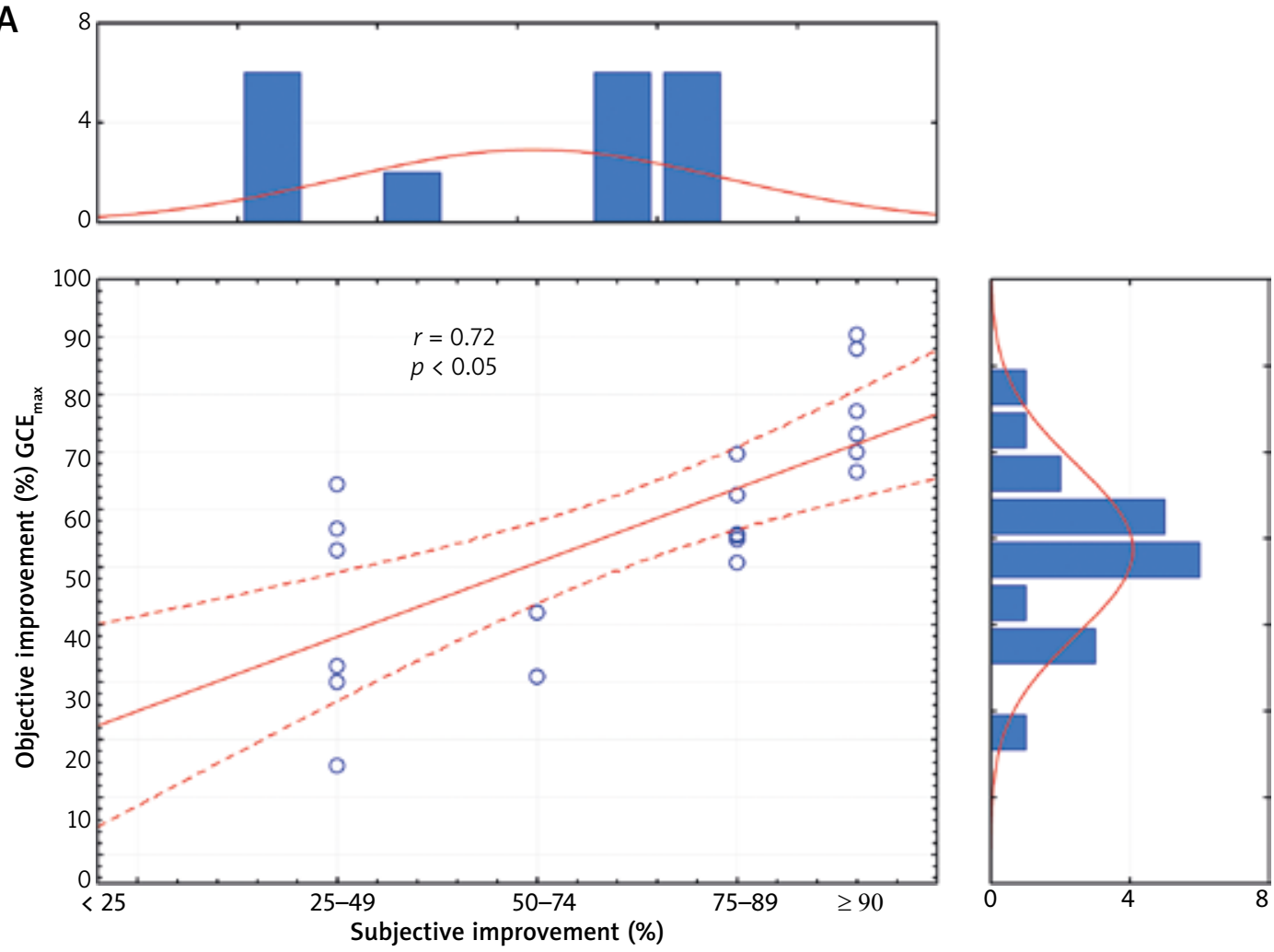

B
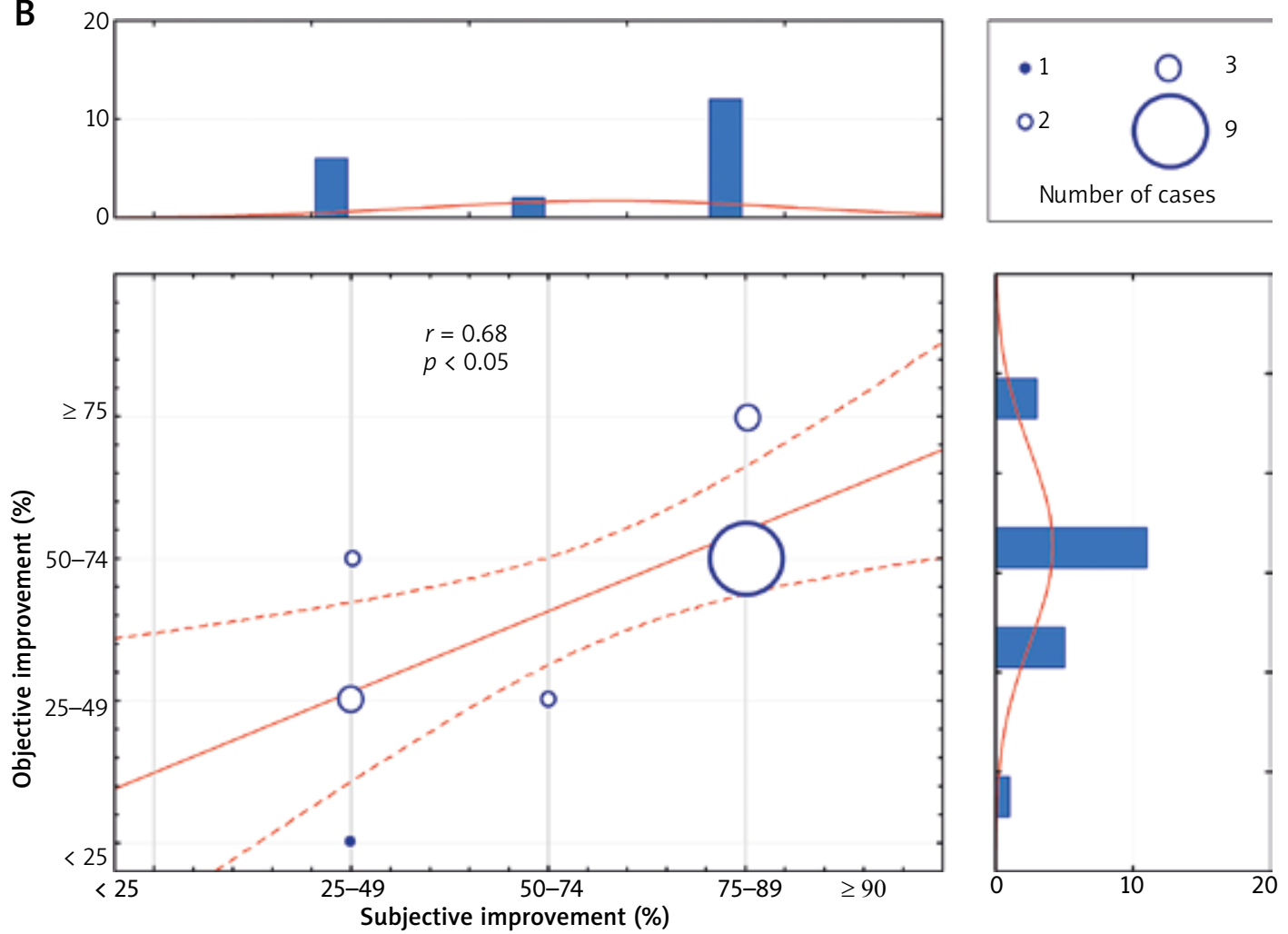

Figure 3. Correlations between objective and subjective methods of improvement assessment. A - Statistically significant positive correlation between $\mathrm{GCE}_{\max }$ values and grade of improvement based on subjective evaluation. Additional rate of $\geq 90 \%$ was added to commonly used thresholds of 25,50 and $75 \%$ for the subjective evaluation. B - Positive correlation between the percentage grade of improvement derived from objective analysis and the percentage rates of improvement based on the subjective evaluation. Relatively low presence of a ' $50-74 \%$ ' grade is seen in the subjective analysis 


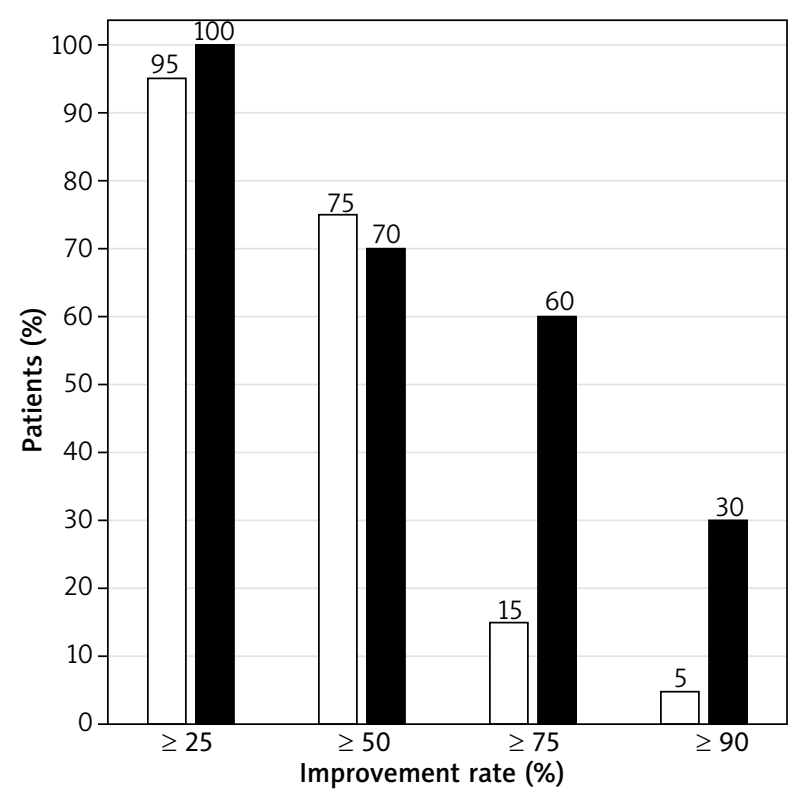

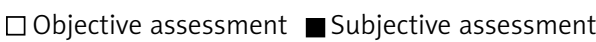

Figure 4. Black bars represent percentage of patients who have achieved maximal improvement of $\geq 25 \%$ (global clearance effect (GCE) 25), $\geq 50 \%$ (GCE 50), $\geq 75 \%$ (GCE 75) and $\geq 90 \%$ (GCE 90) in objective 3D image analysis. Corresponding white bars represent the percentage of patients who have achieved improvement of similar rates assessed in subjective analysis performed by physician $(n=20)$

provement of minimum 25\% (GCE 25) was achieved by all patients but 1 (95\%), and by all patients in subjective analysis. Fifty percentage response rate was achieved by $75 \%$ and $70 \%$ of patients in objective (GCE 50) and subjective methods. The biggest differences were seen in the rates of $75 \%$ and $90 \%$ improvement assessed with the two methods. Only 15\% of patients achieved GCE 75, compared to $60 \%$ of patients with this level of improvement in subjective evaluation. There was a statistically significant correlation between these two methods (Figure $3 \mathrm{~B})$.

Because most studies on treatment efficacy do not use percentage rates of improvement with 25, 50 and $75 \%$ thresholds, but grades of improvement based on ranges, we compared objective and subjective methods using a similar approach (Table 2). There was no statistically significant difference between the two methods, but this analysis reveals a difference between the two types of analysis at the grade of 'significant' (51-75\%) improvement.

\section{Safety}

All patients experienced minor dermal edema lasting usually for up to 4 days and not lasting longer than 7 days. Bruising was also inevitably present and lasted for 7-14 days. Crusting and/or blistering was present
Table 2. Percentages of patients who achieved 'none', 'mild', 'moderate', 'significant' and 'cure' grades of improvement

\begin{tabular}{|c|c|c|c|}
\hline Grade of response & Range (\%) & Objective & Subjective \\
\hline None & $<0$ & 0 & 0 \\
\hline Poor & $1-25$ & $1(5 \%)$ & 0 \\
\hline Moderate & $26-50$ & $5(25 \%)$ & $6(30 \%)$ \\
\hline Significant & $51-75$ & $11(55 \%)$ & $2(10 \%)$ \\
\hline \multirow[t]{2}{*}{ Cured } & $76-100$ & $3(15 \%)$ & $12(60 \%)$ \\
\hline & Sum & 20 (100\%) & 20 (100\%) \\
\hline
\end{tabular}

only focally in a minority of patients. No new scarring was observed except for pre-existing scars after previous procedures in 4 patients. No secondary skin infections were observed.

\section{Discussion}

Our study proved that the large spot $532 \mathrm{~nm}$ laser is effective in the treatment of neck and trunk PWS in Caucasian patients (Figures 1 C-E). Objectively measured rates of improvement were similar to those found for facial PWS $[5,18]$. In these earlier studies we found that in previously untreated facial PWS median GCEmax was $70.4 \%$, which is similar to $69.9 \%$ found in the current study. Similarly, for previously treated patients with facial PWS median GCE max $_{\text {was }}$ 59.09\%, which resembles 55.5\% found for neck and truncal PWS in the present study. We found no difference in $\mathrm{GCE}_{\max }$ between neck and truncal lesions (Figure 2 B). Median number of laser sessions required to gain $\mathrm{GCE}_{\max }$ in our patients was 6 , and this is also comparable to 7 sessions in patients with facial PWS. Thus, all axially localized PWS respond to the large spot $532 \mathrm{~nm}$ laser in the same way. The results obtained here indicate that this method can be judged as a first line treatment not only for facial, but also for neck and truncal PWS together with PDL lasers for patients with fair skin. However, direct comparison in a prospective study would be conclusive.

We introduced the 3D method of PWS color and area evaluation to increase both the accuracy and the objectiveness of treatment outcome measurements. New devices such as the large spot KTP laser presented here and others, e.g. the dual 590/1064 nm laser, PDT with hemoporfin intravenous injection and new medications are being tested for treatment of PWS. Most of the previous and current studies are based on the subjective visual judgment performed by the physician. The common scale used in these reports has a higher threshold point set at 75\%. With the median $\mathrm{GCE}_{\max }$ of about $70 \%$ achieved in untreated patients with the large spot KTP laser, this scale seems to be inadequate. We have proved here that objective assessment proposed by us correlates with commonly used 
subjective methods. However, there are some clear differences. There is a tendency to overestimate the improvement rate performing subjective evaluation in the group of patients who respond with more than $75 \%$ improvement (Figures 1 E, 3, 4, Table 2). It was shown previously that objective analysis of the efficacy of treatment of CM gives slightly worse results than subjective methods [15]. This also supports the use of the new 3D objective analysis for the evaluation of modern highly efficient treatment modalities. Interestingly, there seems to be an opposite phenomenon in the group of patients who responded with less than 50\% improvement (Figures 3, 4, Table 2). There were 3 patients who were judged objectively as improved by more that $50 \%$ but $25-49 \%$ in the subjective method. In general, results obtained in subjective methods are split into $25-49 \%$ improvement and $\geq 75$ with only $10 \%$ judged as $50-75 \%$ improvement (Figure 3, Table 2). In contrast, the distribution of results for the objective method follows a normal distribution pattern with the most common $\mathrm{GCE}_{\max }$ values being about the mean (Figure $3 \mathrm{~A}$ ).

We believe that for the future studies it is better to present results as a percentage rate of improvement rather than as grades. In our previous report we introduced GCE 25, GCE 50, GCE 75 and GCE 90 rates [18]. Physicians and authorities are acquainted with the PASI (Psoriasis Area And Severity Index) 50, PASI 75, PASI 90 terms, and they are currently the main endpoints for the evaluation and comparison of different psoriasis treatment modalities. Results presented in this fashion are clearer than grades. For example, in our study only 10 patients had a significant (51-75\%) response in subjective evaluation (Table 2 ), but $70 \%$ of patients had more than a 50\% response (Figure 4). The latter better reflects the real situation and provides data that can be easily compared with other studies.

We have found the large spot, $532 \mathrm{~nm}$ laser treatment with contact cooling to be safe for treatment of the neck and trunk. All side effects were transient and mild.

\section{Conclusions}

The results of our study indicate that the large spot $532 \mathrm{~nm}$ laser is an effective option for the treatment of neck and truncal PWS and can be used as a first line regimen in patients with type I-III skin phototypes. Objective assessment of the efficacy with 3D image analysis of the neck and trunk is an accurate and reliable method and can be used in future studies to directly compare different methods of PWS treatment.

\section{Conflict of interest}

The authors declare no conflict of interest.

\section{References}

1. Happle R. Capillary malformations: a classification using specific names for specific skin disorders. J Eur Acad Dermatol Venereol 2015; 29: 2295-305.

2. Shirley MD, Tang H, Gallione CJ, et al. Sturge-Weber syndrome and port-wine stains caused by somatic mutation in GNAQ. N Engl J Med 2013; 368: 1971-9.

3. Waelchli R, Aylett SE, Robinson K, et al. New vascular classification of port-wine stains: improving prediction of SturgeWeber risk. Br J Dermatol 2014; 171: 861-7.

4. Jacobs AH, Walton RG. The incidence of birthmarks in the neonate. Pediatrics 1976; 58: 218-22.

5. Kwiek B, Ambroziak M, Osipowicz K, et al. Treatment of previously treated facial capillary malformations: results of single-center retrospective objective 3-dimensional analysis of the efficacy of large spot $532 \mathrm{~nm}$ lasers. Dermatol Surg 2018; 44: 803-13.

6. Zhang B, Zhang TH, Huang Z, et al. Comparison of pulsed dye laser (PDL) and photodynamic therapy (PDT) for treatment of facial port-wine stain (PWS) birthmarks in pediatric patients. Photodiagnosis Photodyn Ther 2014; 11: 491-7.

7. Woo WK, Jasim ZF, Handley JM. Evaluating the efficacy of treatment of resistant port-wine stains with variable-pulse 595-nm pulsed dye and 532-nm Nd:YAG lasers. Dermatol Surg 2004; 30: 158-62.

8. Lorenz S, Scherer K, Wimmershoff MB, et al. Variable pulse frequency-doubled Nd:YAG laser versus flashlamp-pumped pulsed dye laser in the treatment of port wine stains. Acta Derm Venereol 2003; 83: 210-3.

9. Pence B, Aybey B, Ergenekon G. Outcomes of $532 \mathrm{~nm}$ frequency-doubled Nd:YAG laser use in the treatment of portwine stains. Dermatol Surg 2005; 31: 509-17.

10. Chowdhury MM, Harris S, Lanigan SW. Potassium titanyl phosphate laser treatment of resistant port-wine stains. Br J Dermatol 2001; 144: 814-7.

11. Wang BL, Milner H. Laser photocoagulation of port-wine stain blood vessels: three-dimensional monte carlo/finite element simulation with realistic vessel geometry. Lasers Surg Med 2013; 45 39-9.

12. Reddy KK, Brauer JA, Idriss MH, et al. Treatment of port-wine stains with a short pulse width 532-nm Nd:YAG laser. J Drugs Dermatol 2013; 12: 66-71.

13. Al-Janabi MH, Ismaeel Ali NT, Mohamed Al-Sabti KD, et al. A new imaging technique for assessment of the effectiveness of long pulse Nd:YAG $532 \mathrm{~nm}$ laser in treatment of facial port wine stain. J Cosmet Laser Ther 2017; 19: 418-21.

14. Rah DK, Kim SC, Lee KH, et al. Objective evaluation of treatment effects on port-wine stains using L ${ }^{*} a^{*} b^{*}$ color coordinates. Plast Reconstr Surg 2001; 108: 842-7.

15. Szychta P, Al-Nakib K, Anderson W, et al. Quantitative method for evaluation of aesthetic results after laser treatment for birthmarks. Lasers Med Sci 2013; 28: 1567-72.

16. Ion LE, Kavouni A, Scheepers H, Percival N. Objective evaluation of treatment effects on port-wine stains. Plast Reconstr Surg 2002; 110: 712-3.

17. Lister T, Wright P, Chappell P. Spectrophotometers for the clinical assessment of port-wine stain skin lesions: a review. Lasers Med Sci 2010; 25: 449-57.

18. Kwiek B, Rozalski M, Kowalewski C, Ambroziak M. Retrospective single center study of the efficacy of large spot $532 \mathrm{~nm}$ laser for the treatment of facial capillary malformations in 44 patients with the use of three-dimensional image analysis. Lasers Surg Med 2017; 49: 743-9. 